\title{
Impact of contact lens materials on the mfERG response of the human retina
}

\author{
Ana Amorim-de-Sousa $(\mathbb{D} \cdot$ Linda Moreira $\cdot$ Rute Macedo-de-Araújo $(\mathbb{D} \cdot$ \\ André Amorim (D) Jorge Jorge (iD) P Paulo R. Fernandes (D) António Queirós (D) \\ José M. González-Méijome (i)
}

Received: 30 March 2019/ Accepted: 11 September 2019

(C) Springer-Verlag GmbH Germany, part of Springer Nature 2019

\begin{abstract}
Purpose To investigate the effect of different hydrophilic and rigid gas-permeable contact lens (CL) materials on multifocal electroretinography (mfERG).

Methods The mfERG was recorded in 18 healthy subjects with RETI-port/scan $21^{\mathrm{TM}}$ : 11 subjects underwent mfERG recording wearing two different hydrophilic CLs with different water contents in a randomized order (1 silicone hydrogel-Comfilcon A, 48\%EWC, and 1 hydrogel-Omafilcon A, 62\% EWC) and 7 other subjects wore a hydrophobic rigid gas-permeable scleral lens (SL) - Hexafocon A. Control measures were recorded without $\mathrm{CL}$ in both groups. mfERG recordings were performed with a stimulus array pattern of 103-scaled hexagons displayed on a 19-inch RGB monitor at $28 \mathrm{~cm}$ distance at a frame rate of $60 \mathrm{~Hz}$. The amplitude $(\mathrm{nV})$, implicit time and response density $\left(\mathrm{nV} / \mathrm{deg}^{2}\right)$ of the first-order
\end{abstract}

Electronic supplementary material The online version of this article (https://doi.org/10.1007/s10633-019-09722-6) contains supplementary material, which is available to authorized users.

A. Amorim-de-Sousa ( $\square) \cdot$ L. Moreira ·

R. Macedo-de-Araújo · A. Amorim .

J. Jorge · P. R. Fernandes · A. Queirós ·

J. M. González-Méijome

Clinical \& Experimental Optometry Research Lab

(CEORLab), Center of Physics, School of Science,

University of Minho, 4710-057 Gualtar, Braga, Portugal

e-mail: ana.amorim.sousa@gmail.com kernel components N1, P1 and N2 were evaluated for the total mfERG response and for the response averages of 4 quadrants and of 6 successive concentric rings. Subjects were optically corrected for the working distance of ERG display.

Results Hydrophobic material significantly decreased the P1 amplitude of the total mfERG response, at Rings 3, 4 and 6 and Quadrant 4 ( $>53.77 \pm 43.2 \mathrm{nV} ; P \leq 0.050$ ), as well as the total $(-71.59 \pm 50.68 \mathrm{nV}) \quad$ and Ring 6 $(-104.76 \pm 79.88 \mathrm{nV}) \mathrm{N} 2$ amplitude $(P \leq 0.043)$. $\mathrm{N} 1, \mathrm{P} 1$ and $\mathrm{N} 2$ peak times suffered significant changes with both hydrophilic CL $(P \leq 0.050)$. Omafilcon A significantly increased P1 amplitude of Ring 5 and N2 amplitude of Ring 4, when compared to baseline $(52.40 \pm 71.87 \mathrm{nV} ; P=0.036)$ and to Comfilcon A (39.51 $\pm 48.63 \mathrm{nV} ; P=0.023)$, respectively.

Conclusions Hydrophobic CL slightly attenuated the strength of the mfERG signal, especially at the middle to peripheral retinal areas, while hydrophilic CL slightly changed the implicit time of the response. Different hydrophilic CL materials might affect the mfERG response differently. When considering the measurement of mfERG obtained with a CL in place, researchers should bear in mind that some changes can be related to CL material.

Keywords Multifocal electroretinography · Contact lens material - Scleral contact lens · Water content . Electrical conductivity 


\section{Introduction}

Retinal electrical activity can be measured using electroretinography devices. The signal is recorded with an electrode in contact with the ocular surface. In the multifocal electroretinogram (mfERG), the signal from selective parts of the retina can be isolated. This requires a significant number of trials to capture and improve the signal from such a small area with enough signal-to-noise ratio. Any element that can limit the signal that arrives at the active electrode can potentially affect the results, particularly when the signal is relatively small as in the case of the mfERG.

There is an increasing interest to objectively record the electrophysiological response of the visual system when defocused images are presented to the eye through corrective optical devices for myopia control [1] or presbyopia correction [2,3]. The use of contact lenses (CLs), which can be manufactured in a variety of optical designs and can be easily exchanged $[2,4,5]$, makes them ideal optical solutions to change the quality of the image on the retina in a flexible and noninvasive way.

Some active electrodes used for ERG recordings are incorporated in a CL. In fact, it has been reported that ERG CL electrodes, aside from the comfortableness, produce less distortion in the electrical transmission. Early studies have suggested the use of hydrogel materials to create new ERG electrodes to increase the comfort during the ERG recordings. Although these devices are not currently used, those authors report hydrogel materials to be more sensitive to the electrical activity and result in very stable ERG responses when hydration is ensured [6-8].

Different optical devices can be produced with different materials and different electrical conductivity properties, depending on their polymeric structure and water content that can range from 24 up to $78 \%$. Polymers consist mainly of biomaterials containing hydrophilic or hydrophobic monomers. Bordi et al. [9] showed that the electrical conductivity of hydrophilic polymeric structures of poly(ethylene oxide) in aqueous electrolyte solution differed with the polymer concentration but not with the molecular weight of the polymer. Rigid gas-permeable materials are hydrophobic, and water only contacts the CL at the surface where a very thin tear-film layer is formed. Considering the variability in polymeric structure and the possibility that the front surface of a CL can be susceptible to dehydration between blink cycles, it is reasonable to think that wearing a CL while recording an ERG and its material could influence the response recorded.

Considering the above hypothesis, the aim of this study was to test if wearing a contact lens will interfere in the recording of the mfERG response obtained according to the current ISCEV standard. To this extent, different CL hydrophilic materials with different water content and a hydrophobic CL in the mfERG response are evaluated.

\section{Materials and methods}

Study design and subjects

This was a cross-sectional study divided in the evaluation of the impact of two types of hydrophilic CLs and a hydrophobic rigid gas-permeable scleral lens (SL) on the mfERG. The protocol was approved by the Ethics Subcommittee for Health and Life Sciences of the University of Minho and followed the guidelines of the Declaration of Helsinki. After detailed information of the study, all participants gave their signed informed consent.

Eighteen healthy subjects were divided into two groups: one group comprising 11 subjects (7 women) with a mean age of $26.7 \pm 7.11$ years who wore two hydrophilic CLs during mfERG recordings (Comfilcon A, a silicone hydrogel CL, and Omafilcon A, a hydrogel $\mathrm{CL}$ - order randomized for mfERG recording), and the other group comprising 7 subjects (3 women) with a mean age of $32.6 \pm 9.7$ years who wore SL (hydrophobic Hexafocon A material). In both groups, baseline measurements were performed with a naked eye and only the right eyes were evaluated. The spherical equivalent refractive error was $-1.79 \pm 1.32 \mathrm{D}$ (hydrophilic CL group) and $+0.03 \pm 0.70 \mathrm{D}$ (SL group), and refractive astigmatism was inferior to $0.50 \mathrm{D}$ in all subjects. Bestcorrected visual acuity was 0.00LogMAR units or better at baseline and under the different testing conditions for all subjects.

The hydrophilic CL used had a spherical power of $-0.50 \mathrm{D}$, similar diameter $\left(\emptyset_{\text {Comfilcon } \mathrm{A}}=14 \mathrm{~mm}\right.$, $\emptyset_{\text {Omafilcon A }}=14.2 \mathrm{~mm}$ ) and the same base curve of $r_{0}=8.60 \mathrm{~mm}$. Comfilcon A and Omafilcon A have different thicknesses (Comfilcon $A=80 \mu \mathrm{m}$, 
Omafilcon $\mathrm{A}=65 \mu \mathrm{m}$ ) and also differ in oxygen transmissibility (160 DK/t and $28 \mathrm{DK} / \mathrm{t}$, respectively) and water content (48\% and $62 \%$, respectively). The SLs, composed of Hexafocon A (hydrophobic material), were obtained from Procornea Nederland B.V. (Eerbeek, The Netherlands). All subjects from this group were fitted with a SL with the same parameters $\left(\varnothing=16.4 \mathrm{~mm}, \quad r_{0}=8.20 \mathrm{~mm}, \quad\right.$ power $=0.00 \mathrm{D}$, thickness $=402 \mu \mathrm{m}$ and sagittal height $=4673 \mu \mathrm{m}$ ).

\section{mfERG response assessment}

The mfERG technique allows the assessment of many retinal local ERG responses. In this study, the mfERG response was assessed with the RETI-port/scan $21^{\mathrm{TM}}$ (Roland Consult, Brandenburg, Germany).

mfERG recordings were performed on the right eyes of all subjects. In both hydrophilic and hydrophobic CL materials groups, recordings were obtained in a randomized order between all the conditions. Baseline measurements were recorded without a contact lens in place.

Pupils were fully dilated with 2 drops of $1 \%$ Phenylephrine (Davinefrina, DÁVI II), and subjects were optically corrected for the display distance, as recommended by ISCEV standards protocol [10]. The stimulus array consisted of a pattern of 103-scaled hexagons displayed on a 19-inch RGB computer monitor (approximately $37 \mathrm{~cm} \times 30 \mathrm{~cm}$ ) at a working distance of $28 \mathrm{~cm}$ ( 3D vergence). Hexagons flickered at a frame rate of $60 \mathrm{~Hz}$ between white and dark according to an m-sequence. Responses were sampled 16 times per frame (interval of $0.83 \mathrm{~ms}$ ). Each recording lasted $9 \mathrm{~min}$ and $24 \mathrm{~s}$. The size of the 103-scaled hexagonal stimulus array (Fig. 1a) subtended approximately $40^{\circ}-60^{\circ}$ of retinal area (approximately $40^{\circ}-50^{\circ}$ vertically and $50^{\circ}-60^{\circ}$ horizontally). The high and low luminance levels of the stimulus were $220.32 \pm 1.23 \mathrm{~cd} / \mathrm{m}^{2} \quad$ (white) and $1.47 \pm 0.06 \mathrm{~cd} / \mathrm{m}^{2}$ (black), respectively, at $98 \%$ contrast. The monitor illuminance during the measurements at the recording distance $(28 \mathrm{~cm})$ was $152.64 \pm 0.94$ LUX. Considering the mean dilated pupil diameter of $7.84 \pm 054 \mathrm{~mm}$, the mean retinal illuminance was $10,682.63 \pm 1461.31 \mathrm{td}$ for the highest luminance level and $71.23 \pm 9.74$ td for the lowest luminance level. Signals were recorded with a DTL plus electrode placed on the waterline of the lower eyelid (Fig. 2). Patients and signal were continuously checked during the recording through a system inbuilt camera, and if an artifact was seen, the segment of recording was repeated.

The first-order kernel response-first and second negative (N1 and N2, respectively) and first positive (P1) — of the mfERG waveforms (Fig. 1b) was evaluated by retinal quadrants-Quadrants 1 to $4(\mathrm{Q} 1, \mathrm{Q} 2$, Q3 and Q4) - in 6 successive concentric rings scaled from center to periphery: Ring 1 (central macula $0^{\circ}$ $\left.3.61^{\circ}\right)$, Ring $2\left(3.13^{\circ}-10.85^{\circ}\right)$, Ring $3\left(10.85^{\circ}-\right.$ $\left.20.63^{\circ}\right)$, Ring $4\left(20.63^{\circ}-32.46^{\circ}\right)$, Ring $5\left(32.46^{\circ}-\right.$ $\left.46.36^{\circ}\right)$ and Ring $6\left(39.78^{\circ}-58.9^{\circ}\right)$-Fig. 1c. Quadrants 1 to 4 correspond to the inferonasal, superonasal, superotemporal and inferotemporal retina, respectively (see Fig. 1d). From the mfERG signal, the parameters considered were the amplitude (measured between peaks and troughs, in $\mathrm{nV}$ ) of the $\mathrm{P} 1$ and $\mathrm{N} 2$ peaks and the time to peak (or implicit time, measured from the onset of the stimulus to the peak of the component of interest) of N1, P1 and N2 peaks in milliseconds, as represented in Fig. 1b. The changes in response density $\left(\mathrm{nV} / \mathrm{deg}^{2}\right)$ of $\mathrm{N} 1$ and P1 were also evaluated.

\section{Statistical analysis}

As the sample to evaluate the influence of different CL material was not the same for hard and soft materials, data were analyzed and reported separately. All the values are presented as the mean \pm SD differences between baseline and the different CL materials conditions.

Statistical analysis was conducted using SPSS v24.0 (IBM Inc. IL). Normality of data distribution was assessed using the Shapiro-Wilk test. Considering the nature of the data distribution, differences between conditions (CL materials) was assessed using repeated measures Friedman test (non-normally distributed) in hydrophilic CL comparisons and Wilcoxon test for paired comparison in SL condition. The level of statistical significance has been set at $P \leq 0.050$ with $80 \%$ of statistical power. 

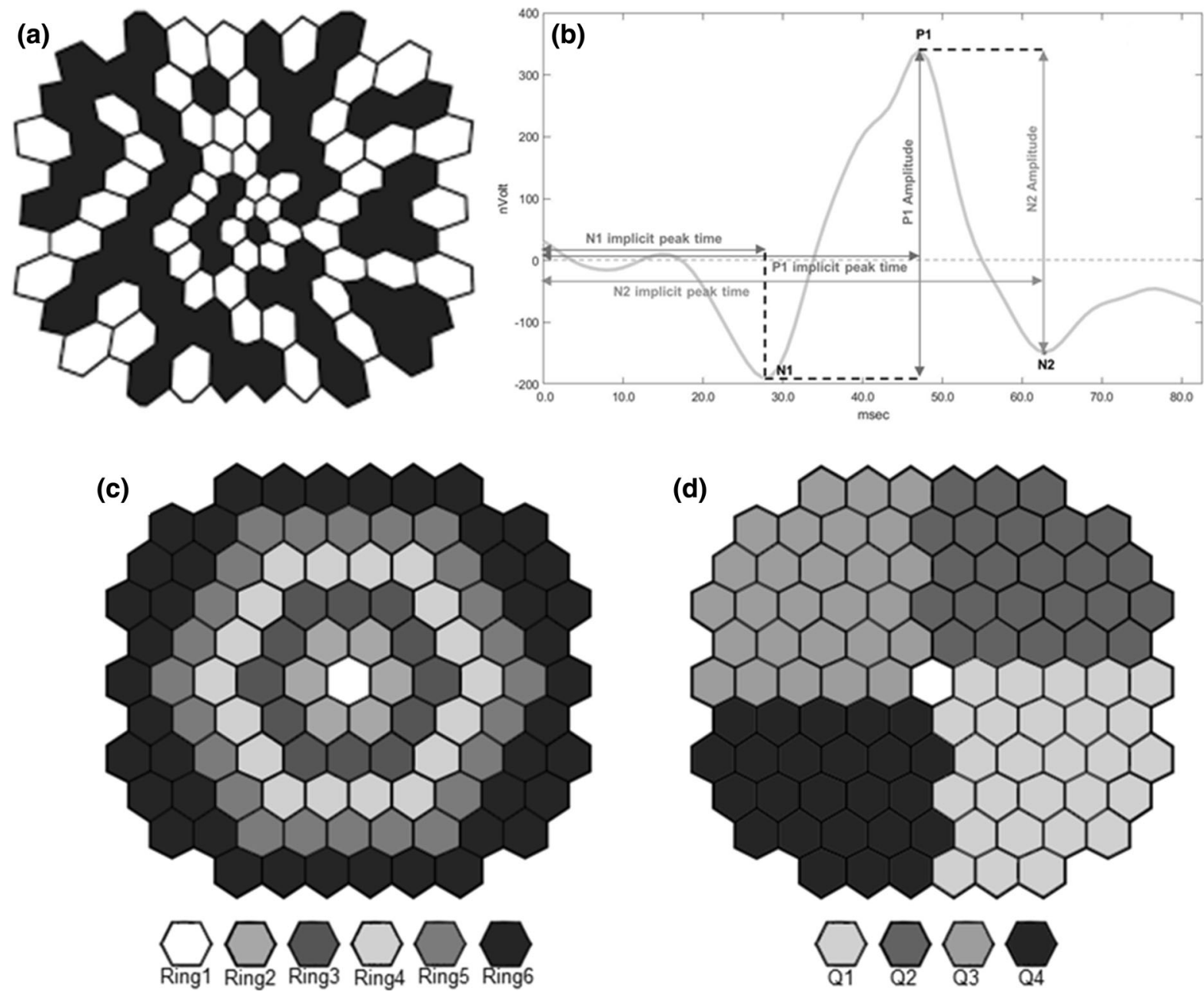

Fig. 1 A stimulus array of 103 hexagons scaled with eccentricity (a) was used to reach the (b) typical waveform of the mfERG obtained for each evaluated area, with three elements of

\section{Results}

Hydrophobic material

Figure 3 represents the graphical variations of the total mfERG response (a) as well as $\mathrm{P} 1$ and $\mathrm{N} 2$ amplitude (b and $\mathrm{c}$, respectively) and N1, P1 and N2 peak times (df) at baseline and scleral lens (SL) material conditions. For the total mfERG response, the mean values of P1 and $\mathrm{N} 2$ amplitudes at baseline were $425.71 \pm 52.75 \mathrm{nV}$ and $376.36 \pm 51.41 \mathrm{nV}$, respectively, and $\quad 353.97 \pm 52.07 \mathrm{nV}$ and $304.77 \pm 49.21 \mathrm{nV}$, respectively, for recordings obtained with a SL in place. The peak implicit time for N1, P1 and N2 components was $25.18 \pm 0.88 \mathrm{~ms}$,

the first-order kernel (N1, P1 and N2)-peak time (ms) and amplitude $(\mathrm{nV})$. The influence of each $\mathrm{CL}$ material was evaluated in (c) 6 concentric rings and (d) 4 quadrants

$45.44 \pm 1.08 \mathrm{~ms}$ and $59.21 \pm 0.82 \mathrm{~ms}$, respectively, for baseline condition, compared to $25.18 \pm 0.54 \mathrm{~ms}$, $44.85 \pm 0.88 \mathrm{~ms}$ and $60.39 \pm 2.92 \mathrm{~ms}$, respectively, with hydrophobic CL material.

In general, the hydrophobic CL material led to a decrease in the amplitudes of all mfERG response components, as shown in Fig. 3a-c. These differences were found to be statistically significant $(P \leq 0.044$, Wilcoxon test) for P1 amplitude of the total mfERG response $(-70.74 \pm 54.52 \mathrm{nV})$, at Rings 3, 4 and 6 $(-55.29 \pm 37.86 \mathrm{nV}, \quad-53.77 \pm 43.29 \mathrm{nV}$ and $-85.46 \pm 55.44 \mathrm{nV}$, respectively) and at Quadrants 1 and $4 \quad(-102.81 \pm 112.46 \mathrm{nV}$ and $-72.54 \pm 40.24 \mathrm{nV}$, respectively). Significant statistical differences were also observed for $\mathrm{N} 2$ 

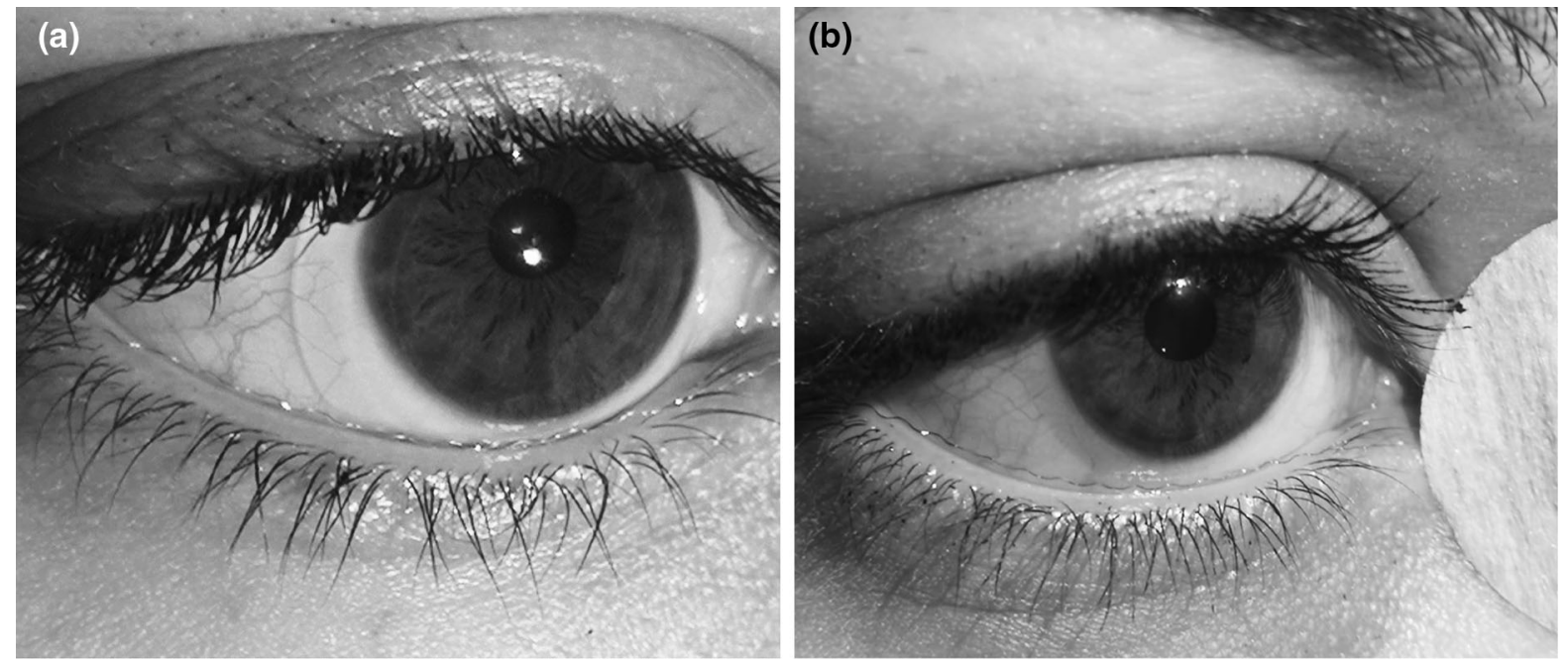

Fig. 2 DTL plus electrode placed on the waterline of the lower eyelid under a (a) hydrophobic scleral lens and a (b) hydrophilic CL (photographs taken without pupil dilation)

amplitude (Fig. 3c) for the total mfERG response $(-71.59 \pm 50.68 \mathrm{nV})$, at the outer ring, Ring 6 $(-104.76 \pm 79.88 \mathrm{nV}), \quad$ and at Quadrant 1 $(-89.14 \pm 90.52 \mathrm{nV}, P=0.043$, Wilcoxon test $)$. For the hydrophobic material, there were no significant changes in the implicit time of any wave peaks (Fig. 3d-f).

Table 1 shows the scaled density regional averages for $\mathrm{N} 1$ and $\mathrm{P} 1$ from the mfERG response (amplitude per squared degree $-n V / \mathrm{deg}^{2}$ ) for all the experimental conditions. The mean $\mathrm{N} 1 \mathrm{nV} / \mathrm{deg}^{2}$ showed an increase with hydrophobic material at all retinal locations, but none of them was statistically significant, except at Quadrant 3 (mean difference of $3.05 \pm 4.16 \mathrm{nV}$ / $\mathrm{deg}^{2}$ ). The opposite is observed for $\mathrm{P} 1 \mathrm{nV} / \mathrm{deg}^{2}$, which decreased with SL, with statistical significance at Ring 6 (mean difference of $6.38 \pm 8.97 \mathrm{nV} / \mathrm{deg}^{2}$ ), Quadrant 1 (mean difference of $8.75 \pm 12.98 \mathrm{nV} / \mathrm{deg}^{2}$ ) and Quadrant 4 (mean difference of $8.30 \pm 11.99 \mathrm{nV}$ / $\mathrm{deg}^{2}$ ), and for the total mfERG response (mean difference of $7.65 \pm 11.10 \mathrm{nV} / \mathrm{deg}^{2}$ ).

\section{Hydrophilic material}

For the hydrophilic material, the total retinal mfERG response and the changes in $\mathrm{P} 1$ and $\mathrm{N} 2$ amplitude as well as N1, P1 and N2 peak times at baseline and with the two hydrophilic CL materials (Comfilcon A and Omafilcon A) are represented in Fig. $4 \mathrm{a}-\mathrm{f}$, respectively. At baseline, the mean values of P1 and N2 amplitudes of the total mfERG were $553.77 \pm 102.75 \mathrm{nV}$ and $429.24 \pm 80.60 \mathrm{nV}$, respectively, and they were $535.83 \pm 140.30 \mathrm{nV}$ and $415.86 \pm 104.81 \mathrm{nV}$ with Comfilcon $\mathrm{A}$ and $602.45 \pm 104.40 \mathrm{nV}$ and $467.65 \pm 92.91 \mathrm{nV}$ with Omafilcon A. The peak implicit times of N1, P1 and $\mathrm{N} 2$ were $27.27 \pm 3.02 \mathrm{~ms}, 47.21 \pm 2.33 \mathrm{~ms}$ and $61.87 \pm 4.11 \mathrm{~ms}$, respectively, at baseline; $28.79 \pm 3.68 \mathrm{~ms}, \quad 48.73 \pm 2.86 \mathrm{~ms} \quad$ and $62.86 \pm 5.67 \mathrm{~ms}$, respectively, with Comfilcon A; and $25.48 \pm 0.82 \mathrm{~ms}, \quad 45.96 \pm 1.17 \mathrm{~ms}$ and $58.74 \pm 1.97 \mathrm{~ms}$, respectively, with Omafilcon A. Figure $4 \mathrm{a}$ shows the total mfERG response that seems, on average, to be higher with Omafilcon $\mathrm{A}$ and reduced with Comfilcon A when compared to baseline.

Regarding peaks' amplitude, the two hydrophilic CL materials did not show significant changes compared to baseline measurements at any topographic area of the retina $(\mathrm{F}(2, \geq 0.677, \quad P \geq 0.134$; $\mathrm{X}^{2}(2) \geq 0.182, P \geq 0.336$ ).

With respect to the implicit time, total mfERG responses differed significantly between the three conditions in $\mathrm{N} 1\left(X^{2}(2)=8.061, P=0.018\right)$ and $\mathrm{P} 1$ $\left(X^{2}(2)=9.243, P=0.010\right)$ peaks. Repeated measures analysis also showed N1 peak time (Fig. 4d) to be significantly different between the three conditions (no CL, Comfilcon A and Omafilcon A) for Rings 2 to 4 and Quadrant $1 \quad(P \leq 0.050)$. The same was observed with P1 peak times (Fig. 4e) for Rings 1, 4 

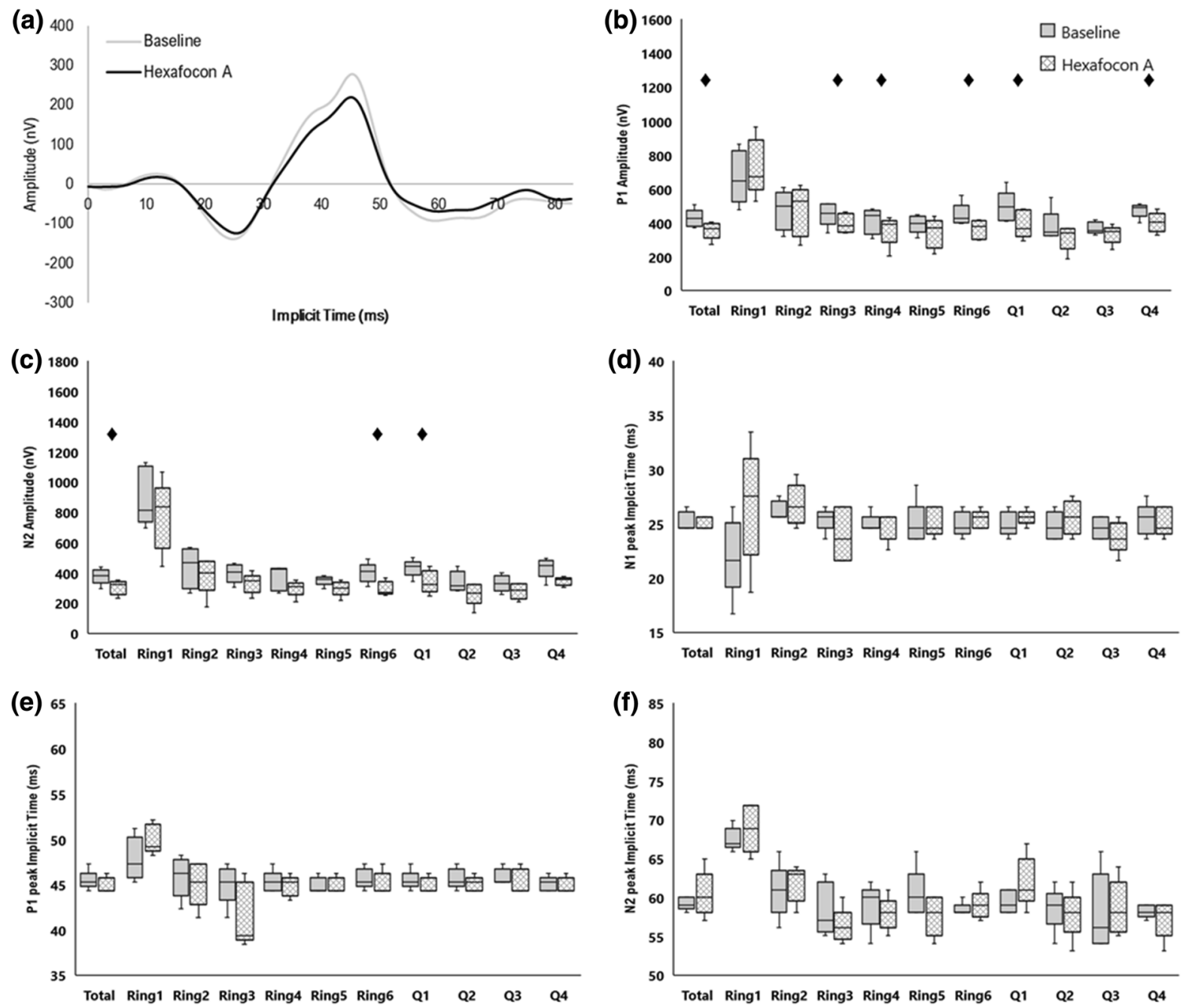

Fig. 3 a Graphical representation of the total retinal mfERG response at baseline (light gray line curve) and with the hydrophobic SL material (black curve). Distribution of P1 and $\mathrm{N} 2$ amplitude (b and $\mathbf{c}$, respectively) and N1, P1 and N2 implicit times (d, e and $\mathbf{f}$, respectively) of the sample group included in

and 6, and Quadrants 3 and $4(P \leq 0.044)$, as well as in $\mathrm{N} 2$ peak time (Fig. 4f) for Quadrant $1\left(X^{2}(2)=7.302\right.$, $P=0.026$, Friedman test). For all the peaks, pairwise comparisons showed that the statistically significant changes observed in implicit times are usually between the two hydrophilic CL materials (Comfilcon A and Omafilcon A, $P \leq 003$ ), except in N1 peak time at Ring 3, where Omafilcon A was smaller than baseline and Comfilcon $\mathrm{A}\left(X^{2}(2)=6.000, P=0.050\right.$, Friedman test). There were no differences in the mean response density $\left(\mathrm{nV} / \mathrm{deg}^{2}\right)$ with both hydrophilic $\mathrm{CL}$

the study of the impact of hydrophobic SL material Hexafocon A (dashed pattern) in the mfERG response, compared to baseline measures without the lens (gray). (Black filled diamond) Statistically significant differences $(P \leq 0.050)$ by paired comparison with Wilcoxon test

materials when compared to baseline values (see Table 1).

\section{Discussion}

The present results confirm that CLs placed on the ocular surface during mfERG recording influence the mfERG response recorded with a DTL electrode. SLs are manufactured in hydrophobic rigid gas-permeable materials. In the present study, the SL material used (Hexafocon A) led to a reduction in amplitude and no 


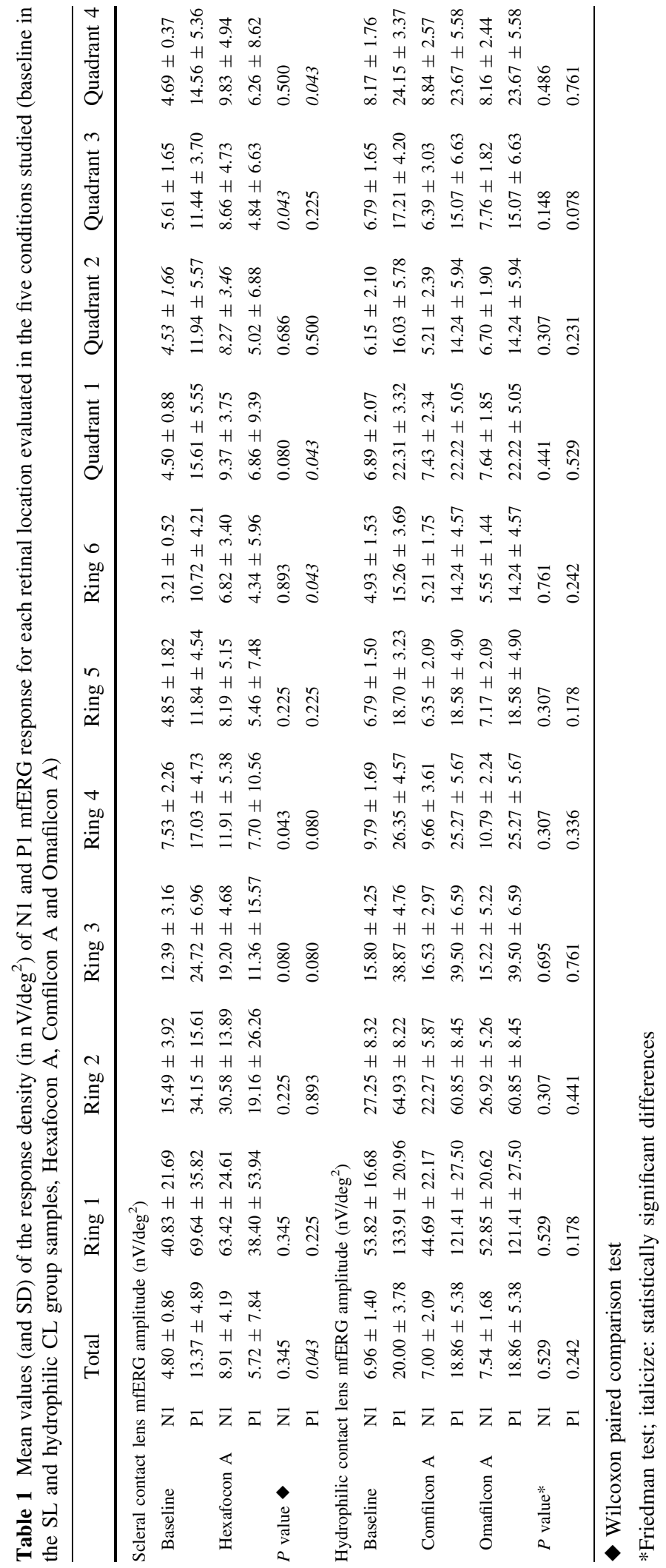



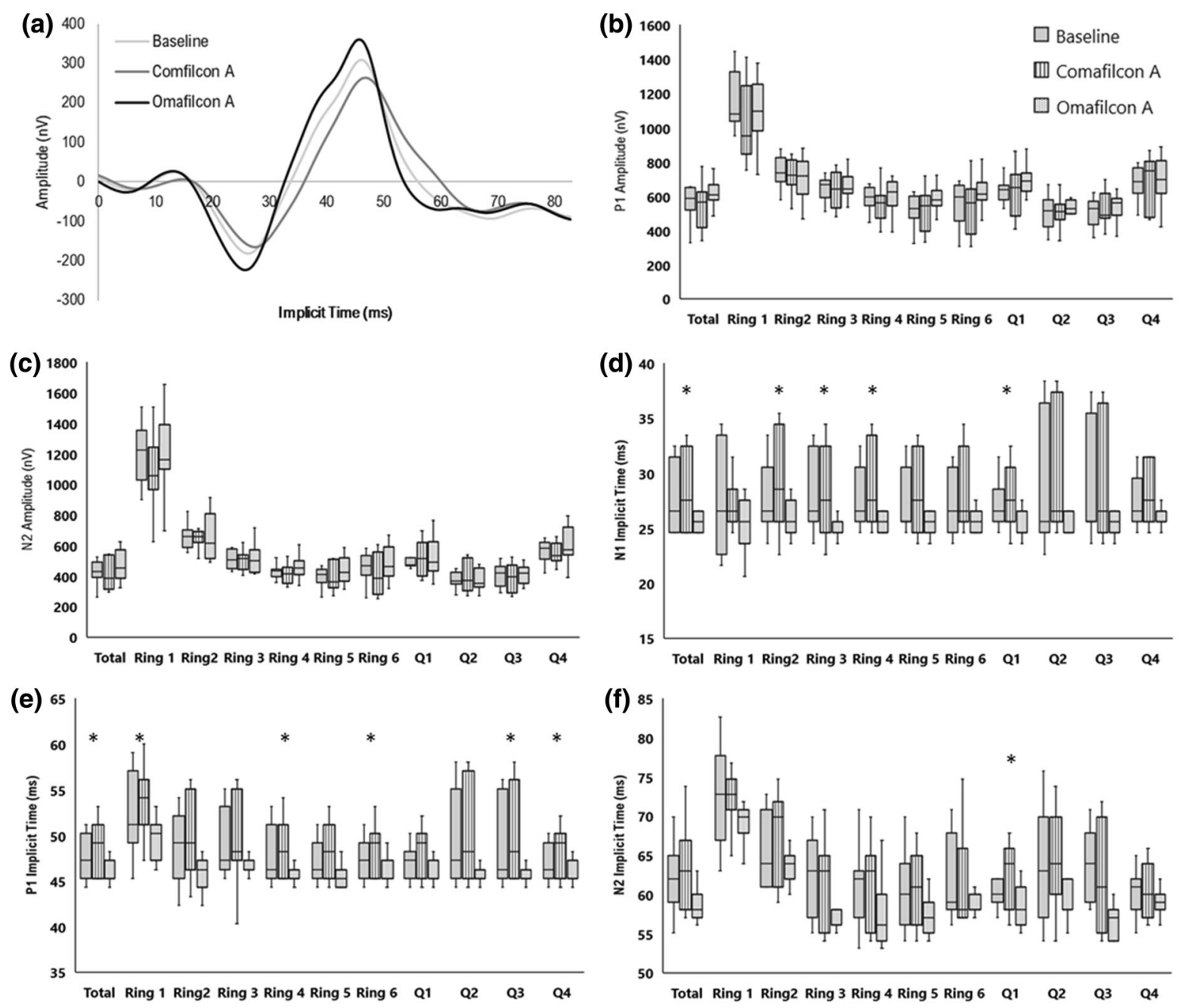

Fig. 4 a Graphical representation of the total retinal mfERG response at baseline (light gray line curve) and with hydrophilic CL (dark gray and black curves, Comfilcon A and Omafilcon A, respectively). Distribution of $\mathrm{P} 1$ and N2 amplitude (b and $\mathbf{c}$, respectively) and N1, P1 and N2 implicit times (d, e and f,

changes in implicit time of the mfERG response (P1 and N2 components) when compared to recording conditions without a contact lens. This suggests that the hydrophobic material may attenuate the strength of the signal (amplitude) that reaches the electrode in contact with the CL polymer. These changes seem to be more significant for middle and peripheral retinal areas compared to central areas. This result suggests that such an attenuation effect might be subtractive. Considering the larger area of those regions, the impact is greater. Therefore, the assessment of central retinal areas might not be significantly influenced by

respectively) of the sample group included in the study of the impact of hydrophilic CL materials Comfilcon A (vertical grating pattern) and Omafilcon A (dotted pattern) in the mfERG response, compared to baseline measures without any lens (gray). *Statistically significant differences by Friedman test

wearing a contact lens, while the assessment of peripheral regions might consider such a potential attenuation effect. With the hydrophilic CL materials, the changes observed were more noticeable in implicit time and differed with the water content of the material. Although the measurements differed more between the two hydrophilic CL materials, differences were also observed compared to baseline where the implicit time of all subjects decreased with Omafilcon $\mathrm{A}$ and increased with Comfilcon $\mathrm{A}$, as shown in Fig. 4d-f. This might suggest that there is a small delay in mfERG signal detection by the active 
electrode placed near the CL material with lower water content (Comfilcon A), compared to the higher water content material (Omafilcon A) that produced a faster response. Additionally, Omafilcon A increased the P1 and N2 amplitudes of the response at more peripheral retinal areas, compared to baseline and Comfilcon A. However, these changes were not statistically significant.

To the best of our knowledge, these phenomena have not been reported yet and deserve some additional considerations. First, the presence of different CL materials at the ocular surface in close proximity to the active electrode seems to have a small effect on both implicit time and response amplitudes of mfERG recordings. This prevents direct comparison with a normative database obtained without CLs. The present study compares the effect of different CL materials which might serve as a reference for other researchers to evaluate the significance of the changes found for their specific applications. Second, the material of CLs seems to play a role in the mfERG response. Hydrophilic material with higher water content (Omafilcon A, 62\%) showed a faster response compared to the hydrophilic material with lower water content (Comfilcon A, 48\%). This phenomenon may be related to differences in electrical conductivity observed in hydrophilic polymers under different conditions of hydration and polymeric composition (hydrogel versus silicone hydrogel, respectively). In 1995, Lopour and Janatová observed that the introduction of hydrogel into a silicone rubber, that is an electrical insulator, turned the silicone rubber into a conductive material. Moreover, as the \% EWC of the silicone hydrogel polymer increases, so does its electrical conductivity [11]. Similarly, Austin and Champeney found that the electrical conductivity of hydrogel polymers could be enhanced by increasing the water content and the porosity [12]. In contrast, an early study of Bordi et al. [9] did not find a relationship between the molecular weight and the electrical conductivity, although a decrease in conductivity was observed as the fractional volume of polymer increased. Third, the effect of the CLs appears to be dependent on the retinal area recorded since more peripheral retinal areas appeared to be more affected by changes rather central areas.

Possible differences could be related to the DTL electrode position. In fact, there are two possible positions for DTL use: placed on the cornea along the lower lid or in the conjunctival fornix. Some studies evaluated the signal differences between the two positions, and they found that when the DTL electrode is positioned in the conjunctival sac, the ERG amplitude is decreased by $20-30 \%$ compared to when the electrode is positioned along the lower lid [13-15]. In the present study, the examiner/operator positioned the DTL electrode on the cornea along the lower eyelid in all subjects, under all the conditions evaluated. Results might be different, if the DTL electrode had been placed in the conjunctival sac, that is more distant to the rim of the CL.

One limitation of the present study was the small sample size. However, considering the consistency of the mfERG results across the $60^{\circ}$ of retinal area, the sample is statistically powerful enough to detect the small differences detected. Another limitation may be the age of the participants. This might explain the differences observed between the two study groups in $\mathrm{P} 1$ and N2 amplitudes as well as N1, P1 and N2 times. In fact, some studies showed a decrease in the amplitude and an increase in peak times with age [16-18]. Although some differences stand out, the mean values and ranges of the mfERG parameters evaluated in the present study at baseline conditions of the two groups (Table 1, Figs. 3 and 4) were similar to those reported in previous studies [18-20]. The differences may be related to different recording systems, as well as age, sample size, and different stimulation paradigms including different m-sequences.

In the present study, the mfERG responses were performed with pupils dilated as recommended by the International Society for Clinical Electrophysiology of Vision (ISCEV) guidelines [10]; the effect of CL material under physiological non-dilated pupil conditions is beyond the scope of the present study.

In summary, practical implications of the present results are relevant for future research protocols involving mfERG measurements in subjects wearing CLs for corrective purposes or to induce changes in the retinal image quality. A control lens made of the same material should be ideally used when CLs are used to change the image quality and mfERG response is obtained with a DTL electrode. Also, when compared to normal databases or other studies performed without CLs, it should be borne in mind that a small change in the implicit time and/or amplitude can be found which may be related to the CL material. 
Depending on the applications, these changes might not be relevant.

Funding This project was supported by the Portuguese Foundation for Science and Technology (FCT) in the framework of projects PTDC/FIS-OPT/0677/2014 and the FCT Strategic Funding UID/FIS/04650/2013, FCT-SFRH/ BPD/92365/2013 and SFRH/BD/136684/2018.

Data availability The datasets generated during and/or analyzed during the current study are available from the corresponding author on reasonable request.

\section{Compliance with ethical standards}

Conflict of interest The authors declare that they do not have any proprietary or financial interest in any of the materials mentioned in this article.

Ethical approval All procedures performed in studies involving human participants were in accordance with the ethical standards of the Ethics Subcommittee for Health and Life Sciences of the University of Minho and followed the 1964 Declaration of Helsinki and its later amendments or comparable ethical standards.

Informed consent Informed consent was obtained from all individual participants included in the study.

Statement of human rights All procedures performed in studies involving human participants were in accordance with the ethical standards of the institutional research committee and with the 1964 Declaration of Helsinki and its later amendments or comparable ethical standards.

Statement on the welfare of animals This article does not contain any studies with animals performed by any of the authors.

\section{References}

1. Ho W-C, Wong O-Y, Chan Y-C, Wong S-W, Kee C-S, Chan HH-L (2012) Sign-dependent changes in retinal electrical activity with positive and negative defocus in the human eye. Vis Res 52:47-53. https://doi.org/10.1016/j. visres.2011.10.017

2. Zeri F, Berchicci M, Naroo SA, Pitzalis S, Di Russo F (2018) Immediate cortical adaptation in visual and non-visual areas functions induced by monovision. J Physiol 596:253-266. https://doi.org/10.1113/JP274896

3. Shimizu K, Ito M (2011) Dissatisfaction after bilateral multifocal intraocular lens implantation: an electrophysiology study. J Refract Surg 27:309-312. https://doi.org/10. 3928/1081597X-20100804-01
4. Imbeau L, Majzoub S, Thillay A, Bonnet-Brilhault F, Pisella P-J, Batty M (2017) Presbyopia compensation: looking for cortical predictors. $\mathrm{Br} \mathrm{J}$ Ophthalmol 101:223-226. https://doi.org/10.1136/bjophthalmol-2015307581

5. El Ameen A, Majzoub S, Pisella P-J (2017) The search for electrophysiological predictors of visual comfort after presbyopia correction with contact lenses. J Fr Ophtalmol 40:257-263. https://doi.org/10.1016/j.jfo.2016.10.014

6. Schoessler JP, Jones R (1975) A new corneal electrode for electroretinography. Vis Res 15:299-301

7. Coupland SG (2006) Principles and practice of clinical electrophysiology of vision, 2nd edn. The MIT Press, Massachusetts

8. Honda Y, Nao-I N, Kim S, Sakaue E, Nambu M (1986) New disposable ERG electrode made of anomalous polyvinyl alcohol gel. Doc Ophthalmol 63:205-207

9. Bordi F, Cametti C, Di Biasio A (1988) Electrical conductivity behavior of poly(ethylene oxide) in aqueous electrolyte solutions. J Phys Chem 92:4772-4777. https://doi. org/10.1021/j100327a042

10. Hood DC, Bach M, Brigell M, Keating D, Kondo M, Lyons JS et al (2012) ISCEV standard for clinical multifocal electroretinography (mfERG) (2011 edition). Doc Ophthalmol 124:1-13. https://doi.org/10.1007/s10633-0119296-8

11. Lopour P, Janatová V (1995) Silicone rubber-hydrogel composites as polymeric biomaterials. Biomaterials 16:633-640. https://doi.org/10.1016/0142-9612(95)938617

12. Austin DTR, Champeney DC (1995) A technique for measuring the alternating current electrical conductivity of hydrogels. J Br Contact Lens Assoc 18:115-118. https://doi. org/10.1016/S0141-7037(95)80022-0

13. Otto T, Bach M (1997) Reproducibility of the pattern electroretinogram. Ophthalmologe 94:217-221

14. Lachapelle P, Benoit J, Little J, Lachapelle B (1993) Recording the oscillatory potentials of the electroretinogram with the DTL electrode. Doc Ophthalmol 83:119-130

15. Kurtenbach A, Kramer S, Strasser T, Zrenner E, Langrová H (2017) The importance of electrode position in visual electrophysiology. Doc Ophthalmol. https://doi.org/10. 1007/s10633-017-9579-9

16. Seiple W, Vajaranant TS, Szlyk JP, Clemens C, Holopigian $\mathrm{K}$, Paliga J et al (2003) Multifocal electroretinography as a function of age: the importance of normative values for older adults. Investig Opthalmol Vis Sci 44:1783. https:// doi.org/10.1167/iovs.02-0518

17. Hansen RM, Moskowitz A, Fulton AB (2009) Multifocal ERG responses in infants. Investig Opthalmol Vis Sci 50:470. https://doi.org/10.1167/iovs.08-2429

18. Molnar AEC, Andreasson SOL, Larsson EKB, Åkerblom HM, Holmström GE (2015) Macular function measured by binocular mfERG and compared with macular structure in healthy children. Doc Ophthalmol 131:169-176. https://doi. org/10.1007/s10633-015-9513-y

19. Dolan FM, Parks S, Keating D, Dutton GN (2006) Wide field multifocal and standard full field electroretinographic 
features of hemi retinal vein occlusion. Doc Ophthalmol 112:43-52. https://doi.org/10.1007/s10633-006-0003-0

20. Ghatak U, Sharma Y, Azad R, Chandra P (2012) Multifocal electroretinogram in normal emmetropic subjects: correlation with optical coherence tomography. Indian J Ophthalmol 60:49. https://doi.org/10.4103/0301-4738.91345
Publisher's Note Springer Nature remains neutral with regard to jurisdictional claims in published maps and institutional affiliations. 